3

4

5

6

7

8

9

10

11

12

13

14

15

16

17

18
SUPPLEMENTAL INFORMATION

MANUSCRIPT ES-2019-03733V

\title{
REPORTED METHANE EMISSIONS FROM ACTIVE OIL AND GAS WELLS IN PENNSYLVANIA, 2014-2018
}

6

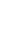

Anthony R. Ingraffea ${ }^{1,2 *}$, Paul A. Wawrzynek ${ }^{3}$, Renee Santoro ${ }^{4}$, Martin Wells $^{5}$

${ }^{1}$ Cornell University, School of Civil and Environmental Engineering, Ithaca, New York 14853 United States

2 PSE Healthy Energy, Inc., Berkeley, California 94612, United States

${ }^{3}$ Fracture Analysis Consultants, Inc, Ithaca, New York 14850 United States

${ }^{4}$ Consultant to Cornell Career Advancement Program for Engineers and Scientists.

${ }^{5}$ Cornell University Ithaca, Department of Statistical Science, New York 14853 United States

17 Pages, 2 Figures, 12 Tables, 3 datasheets

The number of operators has decreased substantially over the 5-year reporting period for all well types except unconventional, Table S1.

Table S1. Number of operators reporting on each type of well.

\begin{tabular}{|l|c|c|}
\hline \multicolumn{1}{|c|}{ Well Type } & $\begin{array}{c}\text { Number of } \\
\text { Operators 2014 }\end{array}$ & $\begin{array}{c}\text { Number of } \\
\text { Operators 2018 }\end{array}$ \\
\hline Conventional Gas & 874 & 450 \\
\hline Conventional Oil & 251 & 163 \\
\hline
\end{tabular}




\begin{tabular}{|l|c|c|}
\hline Conventional Gas and Oil & 205 & 159 \\
\hline Coal-Bed Methane & 12 & 7 \\
\hline Unconventional Gas & 61 & 61 \\
\hline
\end{tabular}

20

21

22

23

24

25

26

27

28

29

30

31

32

33

34

Table S2. Summary of well and well indicator counts for conventional gas wells. Indicators are for production annulus open flow only.

\begin{tabular}{|c|c|c|c|c|c|c|c|}
\hline Year & $\begin{array}{c}\text { \# of } \\
\text { Wells }\end{array}$ & \# CFPD & \# I & \# NA & \# NRM & \# PSIG & $\begin{array}{c}\text { No } \\
\text { Indicator }\end{array}$ \\
\hline $\mathbf{2 0 1 4}$ & 52,088 & 16,327 & 2,271 & 15,089 & 9,148 & 6,488 & 1,388 \\
\hline $\mathbf{2 0 1 5}$ & 51,788 & 17,982 & 1,470 & 11,286 & 10,640 & 6,852 & 2,231 \\
\hline $\mathbf{2 0 1 6}$ & 48,789 & 17,749 & 4,865 & 10,351 & 6,505 & 7,351 & 1,968 \\
\hline $\mathbf{2 0 1 7}$ & 46,631 & 14,389 & 3,042 & 10,984 & 8,472 & 7,686 & 2,148 \\
\hline $\mathbf{2 0 1 8}$ & 52,735 & 13,935 & 2,980 & 11,073 & 13,976 & 8,492 & 2,279 \\
\hline
\end{tabular}

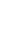

The results in Table S2 show that, for each year, production annulus pressure or open gas flow measurements were made on fewer than one-half of all wells of this type. Measurements were not recorded, were inaccessible, or no indication given on about one-quarter of the wells. Note that in this and similar subsequent tables, the sum of all indicator/no indicator counts will not necessarily equal the number of wells. This is because of errors in data entry by operators. An analysis of whether these indicator counts are compliant with Program directives is given in Section 3.3.

Table S3 lists the operators the conventional gas wells with the ten highest total annular flows for each year or reporting. Note that $\mathrm{mcf} / \mathrm{y}$ is $1,000 \mathrm{cubic}$ feet per year; mmcf/y is $1,000,000 \mathrm{cubic}$ feet per year.

Table S3. Operators of ten conventional gas wells with highest total annular flows.

\begin{tabular}{|c|c|c|c|c|c|c|c|c|c|}
\hline 2014 & & 2015 & & 2016 & & 2017 & & 2018 & \\
\hline Operator & mmcf/y & Operator & mmcf/y & Operator & mmcf/y & Operator & mmcf/y & & \\
\hline
\end{tabular}




\begin{tabular}{|c|c|c|c|c|c|c|c|c|c|}
\hline & & & & & & & & Operator & mmcf/y \\
\hline Exco & 16.7 & Exco & 15.7 & Alliance & 5.5 & Alliance & 14.6 & Alliance & 10.9 \\
\hline Exco & 12.0 & Keyrock & 12.7 & Exotic & 4.4 & Keyrock & 13.2 & Keyrock & 8.6 \\
\hline Exco & 12.0 & Keyrock & 12.4 & Exotic & 3.4 & Alliance & 12.0 & Keyrock & 8.5 \\
\hline Exco & 11.7 & XTO & 10.5 & Alliance & 3.7 & Keyrock & 10.8 & Keyrock & 6.2 \\
\hline Exco & 11.7 & Keyrock & 10.1 & $\begin{array}{c}\text { Indian } \\
\text { Hill } \\
\end{array}$ & 3.7 & Keyrock & 6.7 & Alliance & 5.5 \\
\hline XTO & 10.5 & Exco & 9.7 & $\begin{array}{c}\mathrm{JL} \\
\text { Crawford }\end{array}$ & 3.7 & Keyrock & 6.5 & Alliance & 4.8 \\
\hline Exco & 9.13 & Exco & 9.1 & $\begin{array}{c}\text { Indian } \\
\text { Hill } \\
\end{array}$ & 3.3 & Alliance & 5.8 & Alliance & 4.7 \\
\hline Exco & 9.13 & Exco & 9.1 & $\begin{array}{c}\mathrm{JL} \\
\text { Crawford }\end{array}$ & 3.3 & Alliance & 5.4 & $\begin{array}{c}\text { MDS } \\
\text { Energy }\end{array}$ & 4.4 \\
\hline Exco & 8.4 & Exco & 8.2 & Alliance & 2.9 & Alliance & 5.4 & Alliance & 3.9 \\
\hline Exco & 8.4 & Keyrock & 8.0 & $\begin{array}{c}\text { Indian } \\
\text { Hill } \\
\end{array}$ & 2.9 & $\begin{array}{c}\text { MDS } \\
\text { Energy }\end{array}$ & 5.4 & $\begin{array}{c}\text { JL } \\
\text { Crawford }\end{array}$ & 3.7 \\
\hline $\begin{array}{c}\% \text { of Total } \\
\text { for All } \\
\text { Operators }\end{array}$ & 12.8 & & 13.0 & & 21.8 & & 35.0 & & 36.2 \\
\hline
\end{tabular}

37 Table S4. Summary of well and well indicator counts for conventional oil wells. Indicators 38 are for production annulus open flow only.

\begin{tabular}{|c|c|c|c|c|c|c|c|}
\hline Year & $\begin{array}{c}\text { \# of } \\
\text { Wells }\end{array}$ & \# CFPD & \# I & \# NA & \# NRM & \# PSIG & $\begin{array}{c}\text { No } \\
\text { Indication }\end{array}$ \\
\hline $\mathbf{2 0 1 4}$ & 22,611 & 3,924 & 2,726 & 8,101 & 3,772 & 3,634 & 369 \\
\hline $\mathbf{2 0 1 5}$ & 22,480 & 3,266 & 2,776 & 6,175 & 3,694 & 4,583 & 1,986 \\
\hline $\mathbf{2 0 1 6}$ & 19,640 & 2,192 & 2,666 & 6,099 & 3,819 & 3,286 & 1,578 \\
\hline $\mathbf{2 0 1 7}$ & 18,512 & 2,536 & 1,235 & 5,917 & 3,154 & 4,534 & 1,136 \\
\hline $\mathbf{2 0 1 8}$ & 17,870 & 7,075 & 1,129 & 6,112 & 1,975 & 5,479 & 784 \\
\hline
\end{tabular}

40 Table S4 results show that, for each year, production annulus pressure or open gas flow

41 measurements were made on fewer than one-third of all wells of this type. Measurements were not

42 recorded, were inaccessible, or no indication given on about one-third of the wells. An analysis

43 of whether these indicator counts are compliant with Program directives is given in Section 3.3. 
45 Table S5 lists the operators the conventional oil wells with the ten highest total annular flows for

46 each year or reporting.

47

Table S5. Operators of ten conventional oil wells with highest total annular flows.

\begin{tabular}{|c|c|c|c|c|c|c|c|c|c|}
\hline 2014 & & 2015 & & 2016 & & 2017 & & 2018 & \\
\hline Operator & $\mathrm{mcf} / \mathrm{y}$ & Operator & $\mathrm{mcf} / \mathrm{y}$ & Operator & $\mathrm{mcf} / \mathrm{y}$ & Operator & $\mathrm{mef} / \mathrm{y}$ & Operator & $\mathrm{mef} / \mathrm{y}$ \\
\hline $\begin{array}{c}\text { Pierce\& } \\
\text { Pet. }\end{array}$ & 633.6 & $\begin{array}{c}\text { Pierce\& } \\
\text { Pet. }\end{array}$ & 714.3 & $\begin{array}{c}\text { Pierce\& } \\
\text { Pet. }\end{array}$ & 1,107 & $\begin{array}{c}\text { Pierce\& } \\
\text { Pet. }\end{array}$ & 783.3 & $\begin{array}{l}\text { WB Prod } \\
\text { Mgmt. }\end{array}$ & 3.3 \\
\hline $\begin{array}{c}\text { Pierce\& } \\
\text { Pet. }\end{array}$ & 633.6 & $\begin{array}{c}\text { Pierce\& } \\
\text { Pet. }\end{array}$ & 714.3 & $\begin{array}{c}\text { Pierce\& } \\
\text { Pet. }\end{array}$ & 1,107 & $\begin{array}{c}\text { Pierce\& } \\
\text { Pet. }\end{array}$ & 783.3 & $\begin{array}{l}\text { WB Prod } \\
\text { Mgmt. }\end{array}$ & 2.0 \\
\hline $\begin{array}{c}\text { Pierce\& } \\
\text { Pet. }\end{array}$ & 633.6 & $\begin{array}{c}\text { Pierce\& } \\
\text { Pet. }\end{array}$ & 714.3 & $\begin{array}{c}\text { Pierce\& } \\
\text { Pet. }\end{array}$ & 1,107 & $\begin{array}{c}\text { Pierce\& } \\
\text { Pet. }\end{array}$ & 783.3 & $\begin{array}{c}\text { WB Prod } \\
\text { Mgmt. }\end{array}$ & 1.8 \\
\hline $\begin{array}{l}\text { Pierce\& } \\
\text { Pet. }\end{array}$ & 633.6 & $\begin{array}{l}\text { Pierce\& } \\
\text { Pet. }\end{array}$ & 714.3 & $\begin{array}{l}\text { Pierce\& } \\
\text { Pet. }\end{array}$ & 1,107 & $\begin{array}{l}\text { Pierce\& } \\
\text { Pet. }\end{array}$ & 783.3 & $\begin{array}{l}\text { WB Prod } \\
\text { Mgmt. }\end{array}$ & 1.6 \\
\hline $\begin{array}{l}\text { Pierce\& } \\
\text { Pet. }\end{array}$ & 633.6 & $\begin{array}{c}\text { Pierce\& } \\
\text { Pet. }\end{array}$ & 714.3 & Rannuff & 876.0 & $\begin{array}{l}\text { Pierce\& } \\
\text { Pet. }\end{array}$ & 372.7 & $\begin{array}{l}\text { WB Prod } \\
\text { Mgmt. }\end{array}$ & 1.4 \\
\hline $\begin{array}{c}\text { Pierce\& } \\
\text { Pet. }\end{array}$ & 633.6 & $\begin{array}{c}\text { Pierce\& } \\
\text { Pet. }\end{array}$ & 714.3 & $\begin{array}{c}\text { Pierce\& } \\
\text { Pet. }\end{array}$ & 425.5 & $\begin{array}{c}\text { Pierce\& } \\
\text { Pet. }\end{array}$ & 372.7 & $\begin{array}{l}\text { WB Prod } \\
\text { Mgmt. }\end{array}$ & 1.1 \\
\hline Otter & 584 & $\begin{array}{c}\text { Pierce\& } \\
\text { Pet. } \\
\end{array}$ & 714.3 & $\begin{array}{c}\text { Pierce\& } \\
\text { Pet. }\end{array}$ & 425.5 & $\begin{array}{c}\text { Pierce\& } \\
\text { Pet. }\end{array}$ & 372.7 & $\begin{array}{l}\text { Pierce\& } \\
\text { Pet. }\end{array}$ & 0.82 \\
\hline Otter & 584 & Otter & 456.5 & $\begin{array}{l}\text { Pierce\& } \\
\text { Pet. }\end{array}$ & 425.5 & $\begin{array}{l}\text { Pierce\& } \\
\text { Pet. }\end{array}$ & 372.7 & $\begin{array}{c}\text { Pierce\& } \\
\text { Pet. }\end{array}$ & 0.82 \\
\hline Otter & 584 & Otter & 456.5 & $\begin{array}{c}\text { Pierce\& } \\
\text { Pet. }\end{array}$ & 425.5 & $\begin{array}{c}\text { Pierce\& } \\
\text { Pet. }\end{array}$ & 372.7 & $\begin{array}{c}\text { Pierce\& } \\
\text { Pet. }\end{array}$ & 0.82 \\
\hline Otter & 584 & Otter & 456.5 & $\begin{array}{c}\text { Pierce\& } \\
\text { Pet. }\end{array}$ & 425.5 & $\begin{array}{c}\text { Pierce\& } \\
\text { Pet. }\end{array}$ & 372.7 & $\begin{array}{c}\text { Pierce\& } \\
\text { Pet. }\end{array}$ & 0.82 \\
\hline $\begin{array}{l}\% \text { of Total } \\
\text { for All } \\
\text { Operators }\end{array}$ & 4.1 & & 5.9 & & 9.5 & & 7.7 & & 18.1 \\
\hline
\end{tabular}

49 
51 Table S6. Summary of well and well indicator counts for conventional combined oil/gas wells.

52 Indicators are for production annulus open flow only.

\begin{tabular}{|c|c|c|c|c|c|c|c|}
\hline Year & \# of Wells & \# CFPD & \# I & \# NA & \# NRM & \# PSIG & $\begin{array}{c}\text { No } \\
\text { Indicator }\end{array}$ \\
\hline $\mathbf{2 0 1 4}$ & 6,719 & 962 & 122 & 2,025 & 2,556 & 1,054 & 162 \\
\hline $\mathbf{2 0 1 5}$ & 6,753 & 1,592 & 140 & 1,074 & 2,695 & 1,252 & 315 \\
\hline $\mathbf{2 0 1 6}$ & 6,004 & 1,125 & 209 & 1,110 & 2,638 & 675 & 247 \\
\hline $\mathbf{2 0 1 7}$ & 5,785 & 1,112 & 189 & 851 & 2,738 & 714 & 181 \\
\hline $\mathbf{2 0 1 8}$ & 8,348 & 1,129 & 183 & 883 & 5074 & 963 & 215 \\
\hline
\end{tabular}

53

54 Table S6 results show that, for each year, production annulus pressure or open gas flow

55 measurements were made on fewer than one-third of all wells of this type. Measurements were not

56 recorded, were inaccessible, or no indication given on about one-half of the wells. An analysis of

57 whether these indicator counts are compliant with Program directives is given in Section 3.3.

58

59 Table S7 lists the operators the conventional combined gas and oil wells with the ten highest total

60 annular flows for each year or reporting.

61

62 Table S7. Operators of ten conventional combined gas and oil wells with highest total annular

63 flows.

\begin{tabular}{|c|c|c|c|c|c|c|c|c|c|}
\hline $\mathbf{2 0 1 4}$ & & $\mathbf{2 0 1 5}$ & & $\mathbf{2 0 1 6}$ & & $\mathbf{2 0 1 7}$ & & $\mathbf{2 0 1 8}$ & \\
\hline & & & & & & & & & \\
& & & & & & & & & \\
Operator & $\mathbf{m m c f} / \mathbf{y}$ & Operator & $\mathbf{m m c f} / \mathbf{y}$ & Operator & $\mathbf{m m c f} / \mathbf{y}$ & Operator & $\mathbf{m m c f} \mathbf{y}$ & Operator & $\mathbf{m m c f} / \mathbf{y}$ \\
\hline & & & & & & & & & \\
Exco & 5.5 & Exco & 5.1 & $\begin{array}{c}\text { Pierce } \\
\text { Pet. }\end{array}$ & 1.1 & $\begin{array}{c}\text { Pierce\& } \\
\text { Pet. }\end{array}$ & 2.0 & $\begin{array}{c}\text { Pierce\& } \\
\text { Pet. }\end{array}$ & 1.5 \\
\hline & 5.5 & & 4.4 & Pierce\& & 1.1 & Pierce\& & 2.0 & Pierce\& & 1.5 \\
\hline
\end{tabular}




\begin{tabular}{|c|c|c|c|c|c|c|c|c|c|}
\hline Exco & & Exco & & Pet. & & Pet. & & Pet. & \\
\hline Exco & 4.9 & Exco & 4.0 & $\begin{array}{c}\text { Pierce\& } \\
\text { Pet. }\end{array}$ & 1.1 & $\begin{array}{c}\text { Pierce \& } \\
\text { Pet. }\end{array}$ & 2.0 & $\begin{array}{c}\text { Pierce\& } \\
\text { Pet. }\end{array}$ & 1.5 \\
\hline Exco & 4.8 & Exco & 3.8 & $\begin{array}{c}\text { Pierce\& } \\
\text { Pet. }\end{array}$ & 0.90 & $\begin{array}{c}\text { Pierce\& } \\
\text { Pet. }\end{array}$ & 2.0 & $\begin{array}{c}\text { Pierce\& } \\
\text { Pet. }\end{array}$ & 1.5 \\
\hline Exco & 4.4 & Exco & 3.3 & $\begin{array}{c}\text { Pierce\& } \\
\text { Pet. }\end{array}$ & 0.90 & $\begin{array}{c}\text { Pierce\& } \\
\text { Pet. }\end{array}$ & 2.0 & $\begin{array}{l}\text { Pierce\& } \\
\text { Pet. }\end{array}$ & 1.5 \\
\hline Exco & 4.3 & Exco & 3.1 & Alliance & 0.37 & $\begin{array}{c}\text { Pierce\& } \\
\text { Pet. }\end{array}$ & 2.0 & $\begin{array}{c}\text { Pierce\& } \\
\text { Pet. }\end{array}$ & 1.5 \\
\hline Exco & 4.1 & Exco & 2.4 & Alliance & 0.37 & $\begin{array}{c}\text { Pierce\& } \\
\text { Pet. }\end{array}$ & 0.78 & $\begin{array}{c}\text { Pierce\& } \\
\text { Pet. }\end{array}$ & 1.5 \\
\hline Exco & 3.5 & Exco & 2.2 & Alliance & 0.37 & $\begin{array}{c}\text { Pierce \& } \\
\text { Pet. }\end{array}$ & 0.78 & $\begin{array}{c}\text { Pierce \& } \\
\text { Pet. }\end{array}$ & 0.82 \\
\hline Exco & 3.3 & Exco & 2.2 & $\begin{array}{c}\text { Pierce\& } \\
\text { Pet. }\end{array}$ & 0.28 & $\begin{array}{c}\text { Pierce\& } \\
\text { Pet. }\end{array}$ & 0.78 & $\begin{array}{c}\text { Pierce \& } \\
\text { Pet. }\end{array}$ & 0.82 \\
\hline Exco & 2.9 & Charis & 1.8 & $\begin{array}{c}\text { Pierce\& } \\
\text { Pet. }\end{array}$ & 0.28 & $\begin{array}{c}\text { Pierce\& } \\
\text { Pet. }\end{array}$ & 0.78 & $\begin{array}{c}\text { Pierce\& } \\
\text { Pet. }\end{array}$ & 0.82 \\
\hline $\begin{array}{c}\% \text { of } \\
\text { Total for } \\
\text { All } \\
\text { Operators }\end{array}$ & 43.7 & & 44.0 & & 46.7 & & 62.5 & & 62.0 \\
\hline
\end{tabular}

64

65

66 Table S8. Summary of well and well indicator counts for coal-bed methane wells. Indicators

67 are for production annulus open flow only.

\begin{tabular}{|c|c|c|c|c|c|c|c|}
\hline Year & $\begin{array}{c}\text { \# of } \\
\text { Wells }\end{array}$ & $\begin{array}{c}\# \\
\text { CFPD }\end{array}$ & \# I & \# NA & $\begin{array}{c}\# \\
\text { NRM }\end{array}$ & \# PSIG & $\begin{array}{c}\text { No } \\
\text { Indication }\end{array}$ \\
\hline $\mathbf{2 0 1 4}$ & 685 & 120 & 36 & 411 & 0 & 1 & 11 \\
\hline $\mathbf{2 0 1 5}$ & 666 & 5 & 36 & 486 & 110 & 16 & 13 \\
\hline $\mathbf{2 0 1 6}$ & 415 & 114 & 33 & 234 & 0 & 24 & 10 \\
\hline $\mathbf{2 0 1 7}$ & 630 & 1 & 0 & 375 & 0 & 249 & 5 \\
\hline $\mathbf{2 0 1 8}$ & 620 & 1 & 0 & 491 & 0 & 127 & 1 \\
\hline
\end{tabular}


69 Table S8 results show that, for each year, production annulus pressure or open gas flow

70 measurements were highly variable for CBM wells. Measurements were not recorded, were

71 inaccessible, or no indication given on about one-half of the wells.

72

73 Table S9 lists the operators the coal-bed methane wells with the ten highest total annular flows for

74 each year or reporting. Table S9 clearly shows that emissions from Keyrock wells dominate those

75 from this type of well. However, the Program data offer no explanation for why reported Keyrock

76 well emissions increased over 700-fold between 2014 and 2015, to the highest volume of emissions

77 in any reporting year among all well types; then all Keyrock wells disappear from the data in 2016;

78 then they re-appear in 2017 and produce the third highest volume of emissions among all well

79 types over all reporting years. This anomaly in reporting alone accounts for two-thirds of the

80 decrease in total reported emissions to the Program between 2015 and 2016.

81

82 Table S9. Operators of ten coal-bed methane wells with highest total annular flows.

\begin{tabular}{|c|c|c|c|c|c|c|c|c|c|}
\hline 2014 & & 2015 & & 2016 & & 2017 & & 2018 & \\
\hline Operator & mcf/y & Operator & mmcf/y & Operator & cf $/ \mathbf{y}$ & Operator & mmcf/y & Operator & mmcf/y \\
\hline Keyrock & 50 & Keyrock & 30.5 & - & - & Keyrock & 23.5 & Keyrock & 34.4 \\
\hline $\mathrm{CNX}$ & 37.6 & Keyrock & 26.5 & - & - & Keyrock & 22.6 & Keyrock & 20.4 \\
\hline Keyrock & 31.4 & Keyrock & 18.9 & - & - & Keyrock & 22.4 & Keyrock & 19.5 \\
\hline Keyrock & 29.9 & Keyrock & 18.1 & - & - & Keyrock & 18.8 & Keyrock & 16.0 \\
\hline Keyrock & 23.7 & Keyrock & 18.1 & - & - & Keyrock & 17.4 & Keyrock & 14.1 \\
\hline Keyrock & 19.7 & Keyrock & 17.8 & - & - & Keyrock & 15.5 & Keyrock & 14.1 \\
\hline Keyrock & 18.9 & Keyrock & 17.7 & - & - & Keyrock & 14.5 & Keyrock & 13.2 \\
\hline Keyrock & 18.3 & Keyrock & 16.2 & - & - & Keyrock & 14.1 & Keyrock & 13.2 \\
\hline
\end{tabular}




\begin{tabular}{|c|c|c|c|c|c|c|c|c|c|}
\hline Keyrock & 16.8 & Keyrock & 15.6 & & & Keyrock & 13.1 & Keyrock & 12.8 \\
\hline Keyrock & 16.8 & Keyrock & 15.1 & - & - & Keyrock & 12.4 & Keyrock & 12.1 \\
\hline $\begin{array}{c}\text { \% of Total } \\
\text { for All } \\
\text { Operators }\end{array}$ & 18.9 & & & & & & & & \\
\hline
\end{tabular}

83

84

85

86

87 Table S10 results show that, for each year, production annulus pressure or open gas flow

88

89

90

91 Table S11 lists the operators the unconventional gas wells with the ten highest total annular flows 92 for each year or reporting.

93

94

Table S10. Summary of well and well indicator counts for unconventional gas wells. Indicators are for production annulus open flow only.

\begin{tabular}{|c|c|c|c|c|c|c|c|}
\hline Year & $\begin{array}{c}\text { \# of } \\
\text { Wells }\end{array}$ & $\begin{array}{c}\text { \# } \\
\text { CFPD }\end{array}$ & \# I & \# NA & \# NRM & \# PSIG & $\begin{array}{c}\text { No } \\
\text { Indicator }\end{array}$ \\
\hline $\mathbf{2 0 1 4}$ & 7,800 & 3,016 & 410 & 1,927 & 377 & 1,701 & 286 \\
\hline $\mathbf{2 0 1 5}$ & 8,350 & 3,950 & 333 & 1,265 & 509 & 1,970 & 315 \\
\hline $\mathbf{2 0 1 6}$ & 8,605 & 3,456 & 145 & 1,419 & 938 & 2,635 & 12 \\
\hline $\mathbf{2 0 1 7}$ & 9,458 & 4,305 & 289 & 1,861 & 882 & 2,114 & 7 \\
\hline $\mathbf{2 0 1 8}$ & 10,470 & 6.558 & 557 & 1,670 & 1,237 & 2,510 & 2 \\
\hline
\end{tabular}

measurements were made on about 70 percent of wells. Measurements were not recorded, were inaccessible, or no indication given on over a thousand wells. An analysis of whether these indicator counts are compliant with Program directives is given in Section 3.3.

Table S11. Operators of ten unconventional gas wells with highest total annular flows.

\begin{tabular}{|c|c|c|c|c|c|c|c|c|c|}
\hline $\mathbf{2 0 1 4}$ & & $\mathbf{2 0 1 5}$ & & $\mathbf{2 0 1 6}$ & & $\mathbf{2 0 1 7}$ & & $\mathbf{2 0 1 8}$ & \\
\hline & & & & & & & & & \\
Operator & $\mathbf{m m c f} / \mathbf{y}$ & Operator & $\mathbf{m m c f} / \mathbf{y}$ & Operator & $\mathbf{m m c f} / \mathbf{y}$ & Operator & $\mathbf{m m c f / y}$ & Operator & $\mathbf{m m c f} / \mathbf{y}$ \\
\hline EXCO & 4.0 & EXCO & 4.2 & Cabot & 1.5 & JKLM & 2.3 & Cabot & 3.2 \\
\hline
\end{tabular}




\begin{tabular}{|c|c|c|c|c|c|c|c|c|c|}
\hline Cabot & 3.4 & Vantage & 4.1 & MDS & 1.5 & MDS & 1.5 & JKLM & 3.0 \\
\hline Cabot & 1.9 & EXCO & 4.0 & MDS & 1.5 & Cabot & 1.5 & Cabot & 2.7 \\
\hline Cabot & 1.8 & EXCO & 3.7 & Cabot & 1.4 & $\begin{array}{c}\text { Chesa- } \\
\text { peake }\end{array}$ & 1.4 & JKLM & 2.7 \\
\hline Cabot & 1.3 & MDS & 3.7 & $\begin{array}{c}\text { Chesa- } \\
\text { peake }\end{array}$ & 1.2 & $\begin{array}{c}\text { Chesa- } \\
\text { peake }\end{array}$ & 1.2 & JKLM & 2.2 \\
\hline $\begin{array}{c}\text { Chesa- } \\
\text { peake }\end{array}$ & 1.2 & MDS & 3.7 & Cabot & 1.2 & Cabot & 1.2 & JKLM & 2.2 \\
\hline $\begin{array}{c}\text { Cabot } \\
\text { Cabot }\end{array}$ & 1.2 & MDS & 3.7 & MDS & 1.1 & MDS & 1.2 & JKLM & 2.0 \\
\hline $\begin{array}{c}\text { Cabot } \\
\text { Cabot }\end{array}$ & 0.85 & MDS & 3.7 & Cabot & 1.0 & Cabot & 1.2 & JKLM & 1.9 \\
\hline $\begin{array}{c}\text { \% of } \\
\text { Total for } \\
\begin{array}{c}\text { All } \\
\text { Operators }\end{array}\end{array}$ & 33.5 & 2.8 & Cabot & 1.0 & Cabot & 1.1 & JKLM & 1.8 \\
\hline
\end{tabular}

97 Table S12. Summary of reported incidents of consistent flows outside intermediate and 98 freshwater casings.

\begin{tabular}{|c|c|c|c|c|}
\hline Conventional gas & $\begin{array}{c}\text { Flow } \\
\text { Outside } \\
\text { Intermediate } \\
\text { Casing } \\
\text { (cf/y) }\end{array}$ & $\begin{array}{c}\text { \# wells } \\
\text { with } \\
\text { flow }\end{array}$ & $\begin{array}{c}\text { Flow } \\
\text { Outside } \\
\text { Freshwater } \\
\text { Casing } \\
\text { (cf/y) }\end{array}$ & $\begin{array}{c}\text { \# wells } \\
\text { with } \\
\text { flow }\end{array}$ \\
\hline 2014 & $1,498,325$ & 9 & 825,630 & 31 \\
\hline 2015 & $1,213,990$ & 12 & $1,213,990$ & 4 \\
\hline 2016 & 365 & 1 & 118,990 & 10 \\
\hline 2017 & - & 0 & 1,460 & 4 \\
\hline 2018 & - & 0 & 730 & 2 \\
\hline Conventional oil & & & & \\
\hline 2014 & - & 0 & 3,650 & 1 \\
\hline 2015 & - & 0 & - & 0 \\
\hline 2016 & - & 0 & - & 0 \\
\hline 2017 & - & 0 & - & 0 \\
\hline & - & 0 & - & 0 \\
\hline Unconventional & & & & \\
\hline gas & & & & 2 \\
\hline 2014 & 786,940 & 46 & 3,285 & 1 \\
\hline 2015 & $99,070,490$ & 17 & 730 & \\
\hline
\end{tabular}




\begin{tabular}{|c|c|c|c|c|}
2016 & 79,935 & 6 & - & 0 \\
\hline 2017 & 519,395 & 37 & 20,440 & 10 \\
\hline 2018 & $1,329,695$ & 39 & 3,285 & 4 \\
\hline
\end{tabular}

We use the instructions given to operators in PADEP (2018(e)) to build a logic tree to assess

101

102

103

104

105

106

107

108

109

110

111

112

113

114

115

116

117

118

119

120

121

122

123

124

125

126

127

128

129

130

131

132

133

134

135

136

137

138

139

140

141

compliance in each report to these instructions. We found a significant number of "faults" in the reports, where a fault could mean either an incomplete report or an inconsistency in the application of the instructions. The most common example of a fault is failure to make an entry for either a pressure (PSIG) or flow rate (CPFD) in the production casing annulus. The logic tree shown in Figure S1 was encoded for our assessment of compliance for unconventional gas wells for each of the required four reports for each of five years, 163,030 records.

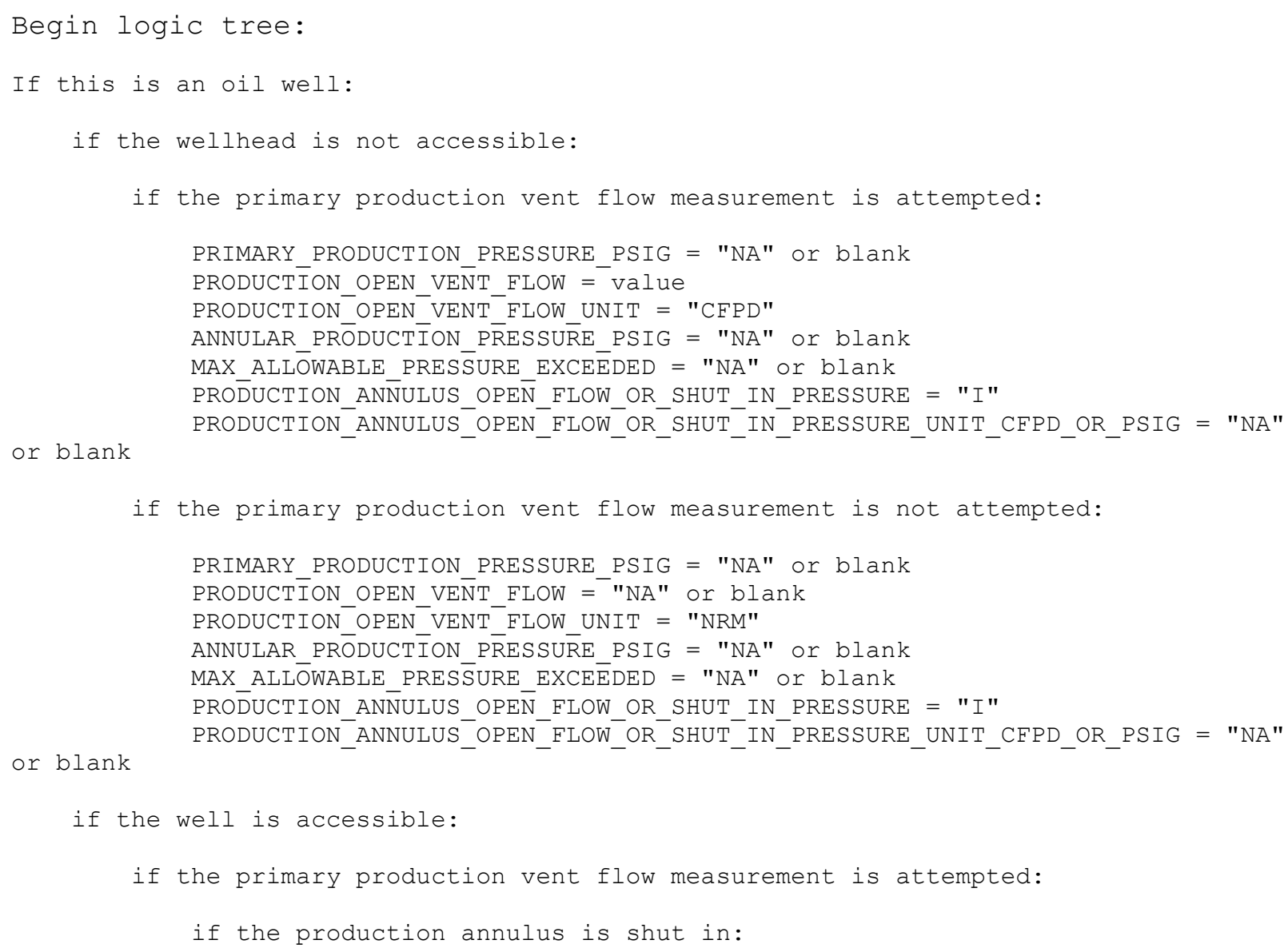


142

143

144

145

146

147

148

149

150

151

152

153

154

155

156

157

158

159

160

161

162

163

164

165

166

167

168

169

170

171

172

173

174

175

176

177

178

179

180

181

182

183

184

185

186

187

188

189

190

191

192

193

194

195

196

197

198

199

200

201

202

203

204
"PSIG"

$=$ "CFPD"

$=" \mathrm{CFPD} "$

if the production annulus open flow measurement is not attempted:

PRIMARY_PRODUCTION_PRESSURE_PSIG = "NA" or blank

PRODUCTION_OPEN VENT_FLOW = value PRODUCTION_OPEN_VENT_FLOW_UNIT $=$ "CFPD"

ANNULAR_PRŌDUCTION_PRESSURE_PSIG = "NA" or blank

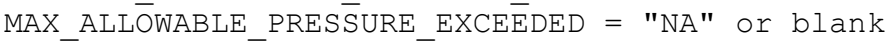
PRODUUCTION ANNULUS OPEN FLOW OR SHUT IN PRESSURE = "NRM" PRODUCTION_ANNULUS_OPEN_FLOW_OR_SHUT_IN_PRESSURE_UNIT_CFPD_OR_PSIG

if the primary production vent flow measurement is not attempted:

if the production annulus is shut in:

PRIMARY_PRODUCTION_PRESSURE_PSIG = "NA" or blank

PRODUCTİON_OPEN_VENTT_FLOW $=$ "NA" or blank

PRODUCTION ${ }^{-}$OPEN ${ }^{-}$VENT ${ }^{-}$FLOW UNIT $=$"NRM"

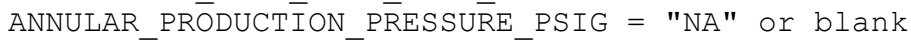

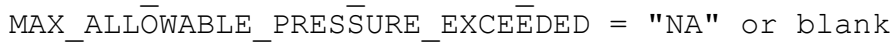

PRODUUCTION_ANNULUS_OPEN_ELOW_OR_SHUT_IN_PRESSURE = value

"PSIG"

PRODUCTION_ANNULUS_OPEN_FLOW_OR_SHUT ${ }^{-}{ }^{-}{ }^{-}{ }^{-}$PRESSURE_UNIT_CFPD_OR_PSIG $=$

if the production annulus is not shut in:

if the production annulus open flow measurement is attempted:

PRIMARY PRODUCTION PRESSURE PSIG = "NA" or blank

PRODUCTİON_OPEN_VEN̄T_FLOW = "NA" or blank

PRODUCTION ${ }^{-}$OPEN ${ }^{-}$VENT ${ }^{-}$FLOW UNIT $=$"NRM"

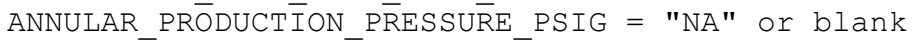

MAX_ALLOWABLE_PRESSTURE_EXCEËDED = "NA" or blank

PRODUUCTION_ANNULUS_OPEN

$=" \mathrm{CFPD} "$

PRODUCTION_ANNULUS_OPEN_FLOW_OR_SHUT_IN_PRESSURE_UNIT_CFPD_OR_PSIG

if the production annulus open flow measurement is not attempted: 
215

216

217

218

219

220

221

222

223

224

225

226

227

228

229

230

231

232

233

234

235

236

237

238

239

240

241

242

243

244

245

246

247

248

249

250

251

252

253

254

255

256

257

258

259

260

261

262

263

264

265

266
PRIMARY PRODUCTION PRESSURE PSIG = "NA" or blank PRODUCTION OPEN VEN̄T FLOW = value PRODUCTION_OPEN VENT_FLOW_UNIT = "CFPD"

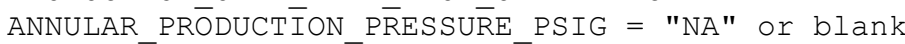
MAX ALLOWABLE PRESSTURE EXCEËDED = "NA" or blank PRODUCTION ANNULUS OPEN FLOW OR SHUT IN PRESSURE = "NRM"

$=$ "CFPD" PRODUCTION_ANNULUS_OPEN_FLOW_OR_SHUT ${ }^{-}{ }^{-}{ }^{-}{ }^{-}{ }^{-}$PRESSURE_UNIT_CFPD_OR_PSIG

if this is a gas or combo well:

if the wellhead is not accessible:

PRIMARY PRODUCTION PRESSURE PSIG = value

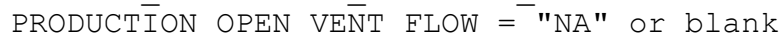
PRODUCTION_OPEN_VENT_ELOW_UNIT = "PSIG" ? ANNULAR_PRODUCTION_PRESSURE_PSIG = "NA" or blank

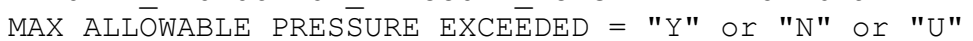

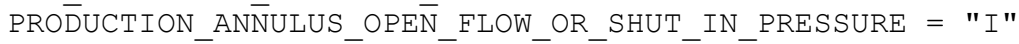
PRODUCTION_ANNULUS_OPEN_FLOW_OR_SHUT_IN_PRESSURE_UNIT_CFPD_OR_PSIG $=$ "NA" or

blank

if the wellhead is accessible:

if the production annulus is shut in:

PRIMARY PRODUCTION PRESSURE PSIG = value PRODUCTION_OPEN_VEN̄T_FLOW $=$ "NA" or blank PRODUCTION OPEN VENT FLOW UNIT = "PSIG"

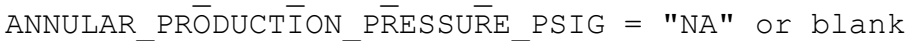
MAX ALLOWABLE PRES $\bar{S} U R E$ EXCEEDED = "NA" or blank PRODUUCTION ANNULUS OPEN FLOW OR SHUT IN PRESSURE = value

"PSIG" PRODUCTION_ANNULUS_OPEN_FLOW_OR_SHUT_IN_PRESSURE_UNIT_CFPD_OR_PSIG =

if the production annulus is not shut in:

if the production annulus open flow measurement is attempted:

PRIMARY PRODUCTION PRESSURE PSIG = value PRODUCTION_OPEN_VENTT_FLOW $=$ "NA" or blank PRODUCTION OPEN VENT FLOW UNIT = "PSIG" ANNULAR PRODUUTT'ON PRESSURE PSIG = "NA" or blank MAX_ALLOWABLE_PRESTSURE_EXCEËDED = "NA" or blank PROD̄UCTION ANNULUS OPEN FLOW OR SHUT IN PRESSURE = value "CFPD" PRODUCTION_ANNULUS_OPEN_FLOW_OR_SHUT_IN_PRESSURE_UNIT_CFPD_OR_PSIG =

if the production annulus open flow measurement is not attempted:

PRIMARY_PRODUCTION_PRESSURE_PSIG = value PRODUCTİON_OPEN_VENTT_FLOW = "NA" or blank PRODUCTION OPEN VENT ${ }^{-}$FLOW UNIT = "PSIG"

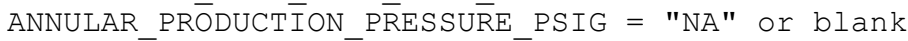
MAX_ALLOWABLE_PRESSURE_EXCEEDED = "NA" or blank

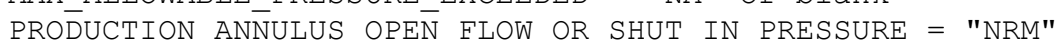
"CFPD" PRODUCTION_ANNULUS_OPEN_FLOW_OR_SHUT ${ }_{-}^{-}$IN_PRESSURE_UNIT_CFPD_OR_PSIG $=$ 
Figure S1. Example logic tree to determine operator compliance with Program directives.

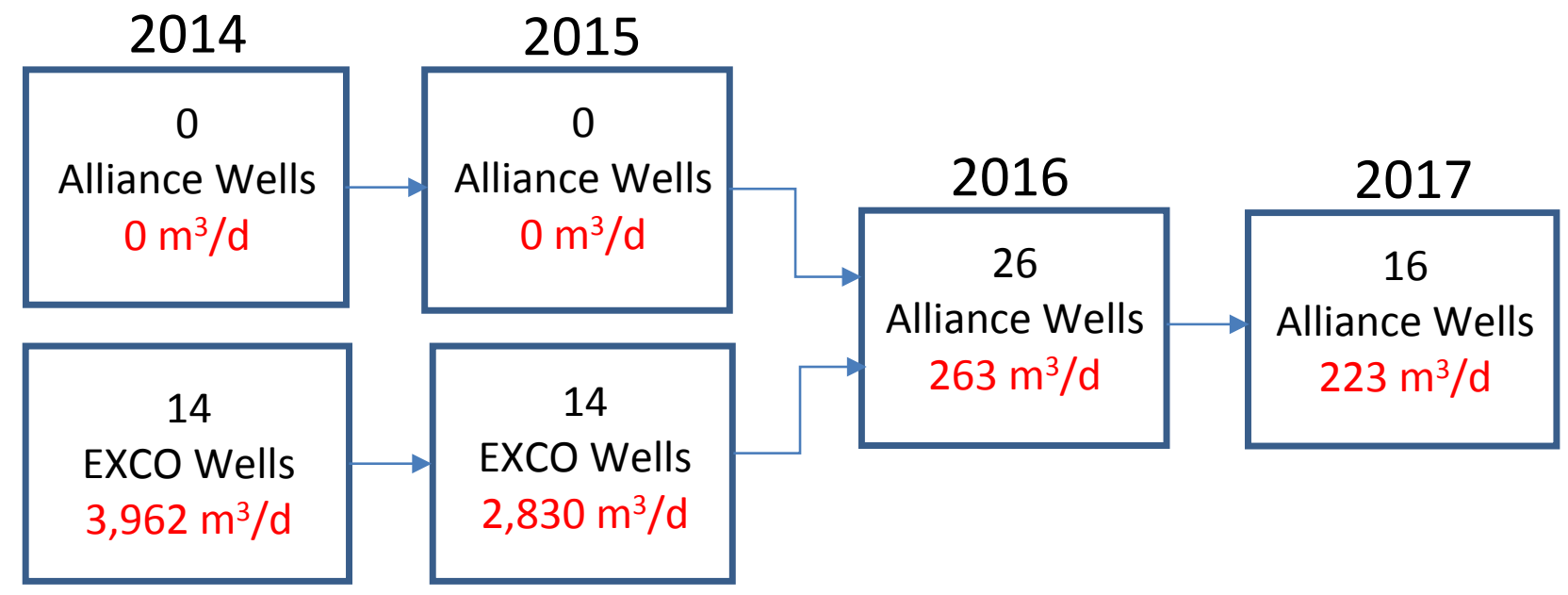

Figure S2. Timeline mapping of actions of two conventional oil and gas well operators leading to the marked reduction in reported emissions between 2015 and 2016 shown in Figure 2. Each box in this figure shows the number of wells for each operator reported under the Program that were in the list of top-emitting 300 wells in that year and their daily emission rate from all flow sources. Numbers in red are before/after measures of emissions from these actions.

\section{PADEP MIA PROGRAM INDICATOR COMPLETE DEFINITIONS (PADEP, 2018b)}

\section{"Production Open Vent Flow}

287 This is the vent flow rate for wells with engineered vents in cases where associated gas is not tied into production. This often applies for oil wells producing inside of surface or coal casing that vent associated gas to comply with $25 \mathrm{~Pa}$. Code Chapter 78, section 78.83(a)(1) or multi-string oil or combination wells that vent production casing gas because either no pipeline or gas market is 
available. It is emphasized that this condition is not a well-construction defect, but rather an engineered vent to regulate pressures and allow liquids production."

293

294

\section{"Production Annulus Open Flow or Shut-in Pressure}

This is the vent flow rate for gas flowing from the production casing annulus in cfpd, if the annulus valve was open and gas was venting to the atmosphere during the inspection. If the production annulus was shut-in during the inspection, the annular pressure in psig is reported. If no flow or pressure is present, the value will be reported as zero (0). If gas is present, but cannot be quantified, NRM should be recorded. Finally, if the production annulus is inaccessible, i.e., subgrade or obstructed by the surface configuration of the well, "I" is entered in this field. Please note that text characters recorded by operators will be stored in the Production Annulus Open Flow or Shut-in Pressure Unit field

All wells with separate production casing strings drilled after February 5, 2011 must be equipped/designed to measure production annulus flow or pressure in order to comply with the monitoring requirements of $25 \mathrm{~Pa}$. Code Chapter 78, section 78.88.

The presence of gas in this case is not necessarily a defect, but instead may be an intentional well design feature. For example, leaving the cement top down on the production casing to vent a shallow gas-bearing zone that is not economical to produce due to low pressure or volume may be one reason for the presence of annular gas. Such a design feature may be important for ensuring that gas is isolated to the wellbore."

\section{"Open Flow Outside Freshwater Casing}

This is the cumulative volume of gas observed outside any freshwater casing string, i.e., coal, surface, or conductor casing. For example, if gas flow is observed in any of these annular spaces, 
314 the operator enters the combined measured or estimated flow rate in cfpd. If gas flow is present,

315 but cannot be quantified, NRM (not readily measurable) is entered. If no gas flow is present outside

316 freshwater casing, the field will either contain a zero (0) or have no value entered. If freshwater

317 casing annular spaces are inaccessible, i.e., subgrade or obstructed by the surface configuration of

318 the well, and fluids were noted in association with some other well component, "I" is entered in

319 this field. "I" may also be found in this field if data were derived from Form A or B. Please note

320 that text characters recorded by operators will be stored in the Open Flow Outside Freshwater

321 Casing Unit field. Any gas sourced from below a freshwater casing shoe that is flowing through

322 the fresh groundwater interval is prohibited by $25 \mathrm{~Pa}$. Code Chapter 78, section 78.81(a)(3). $25 \mathrm{~Pa}$.

323 Code Chapter 78, section 78.85(a)(5) also prohibits gas flow outside surface or coal casing 324 strings."

\section{5 "Open Flow Outside Intermediate Casing}

326 This is the cumulative volume of gas observed outside any intermediate casing strings. If gas flow

327 is observed in any of these annular spaces, the operator enters the measured or estimated flow rate

328 in cfpd; or if gas flow is present but cannot be quantified, NRM (not readily measurable) is entered.

329 If no gas flow is present outside intermediate casing, the field will either contain a zero (0) or have

330 no value entered. If intermediate casing annular spaces are inaccessible, i.e., subgrade or

331 obstructed by the surface configuration of the well, and fluids were noted in association with some

332 other well component, "I" is entered in this field. "I" may also be found in this field if data were

333 derived from Form A or B. Please note that text characters recorded by operators will be stored in

334 the Open Flow Outside Intermediate Casing Unit field.” 